\title{
Zingiber officinale Roscoe rhizome extract alleviates neuropathic pain by inhibiting neuroinflammation in mice
}

\author{
Vittoria Borgonetti ${ }^{\mathrm{a}}$, Paolo Governa ${ }^{\mathrm{b}}$, Marco Biagi ${ }^{\mathrm{c}}$, Federica Pellati ${ }^{\mathrm{d}}$, Nicoletta Galeotti ${ }^{\mathrm{a}, *}$ \\ ${ }^{a}$ Department of Neuroscience, Psychology, Drug Research and Child Health (NEUROFARBA), Section of Pharmacology, University of Florence, Viale G. Pieraccini 6, \\ 50139 Florence, Italy, \\ ${ }^{\mathrm{b}}$ Department of Biotechnology, Chemistry and Pharmacy - Department of Excellence 2018-2022, University of Siena, Via Aldo Moro 2, 53100 Siena, Italy \\ ${ }^{\mathrm{c}}$ Department of Physical Sciences, Earth and Environment, University of Siena, Strada Laterina 8, 53100 Siena, Italy \\ ${ }^{\mathrm{d}}$ Department of Life Science, University of Modena and Reggio Emilia, Via G. Campi 103, 41125 Modena, Italy
}

\section{A R T I C L E I N F O}

\section{Keywords:}

Zingiber officinale roscoe

Neuropathic pain

Microglia

MAPK

HDAC

Neuroinflammation

\begin{abstract}
A B S T R A C T
Background: Current therapies for neuropathic pain are generally symptomatic and possess several side effects, limiting their prolonged usage.

Hypothesis/Purpose: Thus, it is urgent to develop novel and safe candidates for the management of this chronical condition. For this purpose, we investigated the analgesic effect of a standardized extract from Zingiber officinale Roscoe rhizomes (ZOE) obtained by $\mathrm{CO}_{2}$ supercritical extraction, in a mice model of peripheral neuropathy. We also explored the mechanism of action of ZOE and its main constituents using an in vitro model of neuroinflammation.

Methods: Peripheral mono-neuropathy was induced in mice, by spared nerve injury (SNI). The analgesic effect of ZOE after oral administration was assessed by measuring mechanical and thermal allodynia in SNI mice. The mechanism of action of ZOE and its main constituents were investigated using spinal cords samples and in an in vitro model of neuroinflammation by ELISA, western blotting and immunofluorescence techniques.

Results: Oral administration of ZOE $200 \mathrm{mg} \mathrm{kg}^{-1}$ ameliorated mechanical and thermal allodynia in SNI mice, with a rapid and a long-lasting effect. ZOE did not alter locomotor activity. In BV2 cells and spinal cord samples, ZOE, 6-gingerol and 6-shogaol reduced pERK levels, whereas ZOE and terpene fraction reduced HDAC1 protein levels, inhibited NF- $\mathrm{\kappa B}$ signalling activation and decreased IL-1 $\beta$, TNF- $\alpha$ and IL- 6 release. ZOE and each tested constituent had a positive effect on inflammation-impaired SH-SY5Y cell viability.

Conclusions: The oral administration of ZOE attenuated SNI-induced neuropathic pain symptoms by reducing spinal neuroinflammation, suggesting ZOE as a novel and interesting candidate for the management of neuropathic pain.
\end{abstract}

\footnotetext{
Abbreviations: ANOVA, analysis of variance; ARRIVE, animal research: reporting of in vivo experiments; CMC, sodium carboxymethyl cellulose; CNS, central nervous

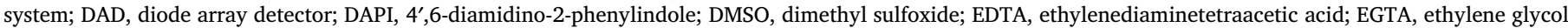

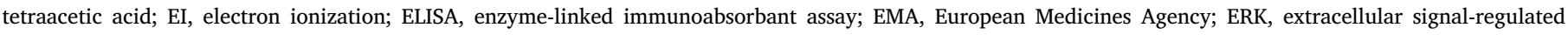

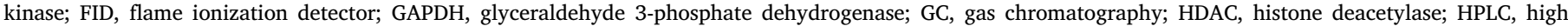

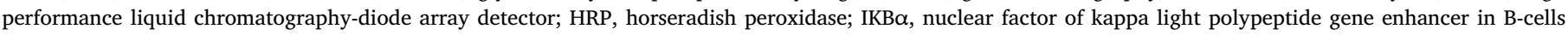

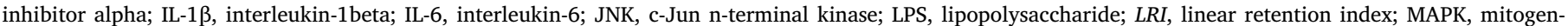

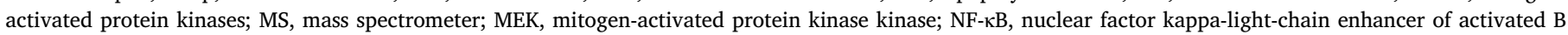

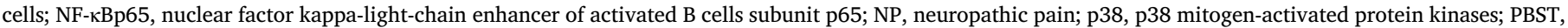

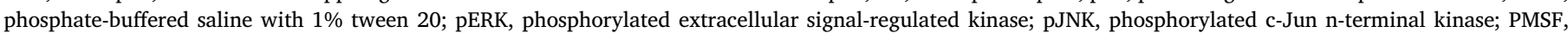

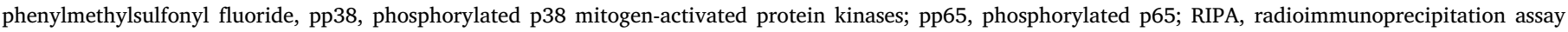

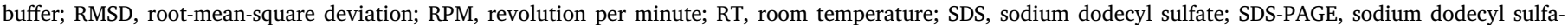

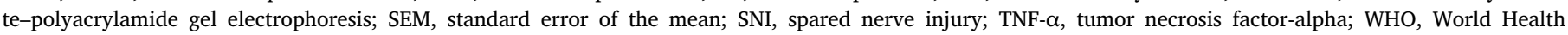
Organization; ZOE, standardized Zingiber officinale Roscoe rhizome extract; ZTE, Zingiber officinale terpenoid-enriched extract

* Corresponding author.

E-mail address: nicoletta.galeotti@unifi.it (N. Galeotti).
} 


\section{Introduction}

Neuropathic pain (NP) is a multifactorial condition caused by a lesion or disease of the somatosensory system, involving different pathophysiological mechanisms. Patients with NP exhibit common signs of hypersensitivity to pain, including mechanical allodynia, a painful sensation caused by innocuous stimuli, and hyperalgesia, an increase of sensitivity to pain (Jensen and Finnerup, 2014). Current therapies for the management of NP are generally symptomatic and rarely focused on the actual causes. Furthermore, these medications are characterized by several well-known side effects, which limit their prolonged use (Jensen and Finnerup, 2014). Thus, it is urgent to develop novel and safe candidates for the management of this chronic condition.

According to the World Health Organization (WHO), an increasing number of patients support the use of herbal treatments because they are generally considered safer than conventional medicine (Singh et al., 2017). Recently, an important biological role has been attributed to curcumin, a phenolic compound derived from Curcuma longa L. (Zingiberaceae) rhizome (turmeric), in the management of pain (Sun et al., 2018). However, even though different strategies have been studied to improve the absorption of curcumin (i.e. nanocrystals, emulsions, liposomes, nanogels), its low bioavailability limits its application as a therapeutic agent. Moreover, the lack of information about the safety profile of the novel curcumin formulation, together with their expensive costs, raised the need to search for other herbal species with similar activity, but with a more favourable bioavailability profile (Zhao et al., 2019).

Zingiber officinale Roscoe (Zingiberaceae), commonly known as ginger, is an Asian-native species belonging to the same family of turmeric and it is widely used as a spice. Ginger is enlisted in many official pharmacopoeias of different countries, including European Pharmacopoeia $9^{\text {th }}$ ed. The dried rhizome, the part of the plant with biological activities, contains a complex mixture of bioactive compounds with the essential oil and oleoresin representing the most abundant substances of the phytocomplex. The non-volatile components, to which the characteristic pungent taste is attributed, include gingerols, shogaols, paradols and zingerone (Semwal et al., 2015). Several studies have proved that $Z$. officinale rhizome possesses a broad range of pharmacological actions and it can be efficiently used for treating nausea and vomiting, gastrointestinal problems, hyperglycemia, dysmenorrhea, inflammatory disorders and pain (Forouzanfar and Hosseinzadeh, 2018). In spite of the similarity between ginger and turmeric chemical composition, ginger and its main constituents have been shown to possess an optimal pharmacokinetic profile, thus, overcoming one of the main limitation for the therapeutic use of turmeric. Indeed, it has been recently observed that ginger administered via p.o is neuroprotective in different in vivo models of neurological disorders (Choi et al., 2018), supporting its capability to reach the central nervous system (CNS). Importantly, according to the European Medicines Agency (EMA), safety data for ginger are generally positive and only few minor adverse effects have been observed (EMA, 2012). Thus, the aim of our study was to investigate the possible analgesic effect of a standardized $Z$. officinale rhizomes extract (ZOE) obtained by $\mathrm{CO}_{2}$ supercritical extraction, in a mice model of peripheral mononeuropathy, i.e. the spared nerve injury (SNI), to further elucidate exploring the mechanism of action of ZOE and its main constituents in an in vitro model of neuroinflammation.

\section{Materials and methods}

Chemicals

ZOE, obtained by supercritical $\mathrm{CO}_{2}$ extraction, and standardized to contain $24.73 \%$ total gingerols and $3.03 \%$ total shogaols, was kindly provided by INDENA S.p.A. (Milan, Italy), batch number 46349. 6gingerol (GIN) was purchased from Sigma-Aldrich (Milan, Italy) and 6- shogaol (SHO) was purchased from Extrasynthese (Genay, France). GC grade $n$-Hexane was from J.T. Baker (Milan, Italy). U0126 was purchased from Calbiochem (Milan, Italy). Bacterial lipopolysaccharide (LPS) from Gram- (Salmonella enteridis) was purchased from SigmaAldrich. All the analytical standards were from Sigma Aldrich.

\section{Quantification of 6-gingerol and 6-shogaol by HPLC-DAD}

ZOE was diluted 100 -fold in $96 \% \mathrm{v} / \mathrm{v}$ ethanol and filtered through $0.45 \mu \mathrm{m}$ membrane before HPLC-DAD analysis. A Shimadzu Prominence LC 2030 3D instrument, equipped with a Bondapak ${ }^{\circledR} C_{18}$ column $(300 \times 3.9 \mathrm{~mm}, 10 \mu \mathrm{m}, 125 \AA$, Waters Corporation, Milford, MA, USA) as the stationary phase, was used. The mobile phase was composed of water with $0.5 \%$ formic acid (A) and acetonitrile with $0.1 \%$ formic acid (B), under the following gradient: from $50 \%$ A to $45 \%$ A in $8 \mathrm{~min}$, followed by an isocratic phase until $10 \mathrm{~min}$. The flow rate was $0.9 \mathrm{ml} / \mathrm{min}$ and the injection volume was $10 \mu \mathrm{l}$.

The absorbance was recorded at $280 \mathrm{~nm}$ and the quantification of GIN and SHO was performed according to the calibration curves obtained using analytical grade standards.

\section{Qualitative and semi-quantitative analysis of ZOE volatile compounds}

Volatile compounds in ZOE were analysed by means of gas chromatography coupled with a flame ionization detector (GC-FID) and with a mass spectrometer (GC-MS), using an Agilent Technologies (Waldbronn, Germany) 7820A instrument. For GC-MS, a 5975C network mass spectrometer (Agilent Technologies) was used.

The following conditions were applied for both GC-FID and GC-MS: compounds were separated on an Agilent Technologies HP-5 crosslinked poly-5\% diphenyl-95\% dimethyl polysiloxane $(30 \mathrm{~m} \times 0.32 \mathrm{~mm}$ i.d., $0.25 \mu \mathrm{m}$ film thickness) capillary column. The injection volume was $0.1 \mu \mathrm{l}$ with a split ratio of 1:20 and 1:40 in GC-FID and GC-MS analysis, respectively. The column temperature was initially set at 45 ${ }^{\circ} \mathrm{C}$, then increased to $100{ }^{\circ} \mathrm{C}$ at a rate of $2{ }^{\circ} \mathrm{C} / \mathrm{min}$ up, then raised to 250 ${ }^{\circ} \mathrm{C}$ at a rate of $5{ }^{\circ} \mathrm{C} / \mathrm{min}$, which was held for $5 \mathrm{~min}$. Helium was used as the carrier gas at a flow rate of $1.0 \mathrm{ml} / \mathrm{min}$. The injector and FID detector temperature were set at 250 and $300{ }^{\circ} \mathrm{C}$, respectively. Concerning the MS detector, the transfer line and ion-source temperature were 280 , and $230{ }^{\circ} \mathrm{C}$, respectively. MS detection was performed with electron ionization (EI) at $70 \mathrm{eV}$, operating in the full-scan acquisition mode in the $m / z$ range $40-400$. The sample was diluted $1: 20(\mathrm{v} / \mathrm{v})$ with $n$ hexane before GC-FID and GC-MS analyses. The analyses were performed in triplicate.

Compounds were identified by comparing the retention times of the chromatographic peaks with those of authentic reference standards run under the same conditions and by comparing the experimental linear retention index $(L R I)$ values, calculated from a mixture of $n$-alkanes $\left(\mathrm{C}_{8}-\mathrm{C}_{40}\right)$ in $n$-hexane and injected under the same conditions as those previously described in the literature (Adams, 2007). Peak enrichment by co-injection with authentic reference compounds was also carried out. Comparison of the MS-fragmentation pattern of the target analytes with those of pure components was performed. A mass-spectrum database search was performed by using the National Institute of Standards and Technology (NIST, Gaithersburg, MD, USA) mass-spectral database (version 1.4). The percentage relative amount of individual components was expressed as percent peak area relative to total peak area.

\section{Terpenoid-enriched fraction extraction and quantification}

The extraction of the terpenoid-enriched fraction (ZTE) was performed as previously described (Ferguson, 1956). ZOE (100 mg) was soaked in $10 \mathrm{ml}$ of $96 \% \mathrm{v} / \mathrm{v}$ ethanol for $24 \mathrm{~h}$. The extract was filtered and then extracted with $10 \mathrm{ml}$ of petroleum ether, using a separating funnel. The petroleum ether extract was separated in pre-weighed 15 
$\mathrm{ml}$ tubes and evaporated. The yield (\%) of ZTE content was calculated using the following formula:

$($ wi-wf/wi $\times 100)$

where wi is the initial weight of ZOE and wf is the final weight of the petroleum ether extract after evaporation.

Animals

CD1 male mice (20-22 g) from the Envigo (Varese, Italy) were used. Mice were randomly assigned to standard cages, with four to five animals per cage. The cages were placed in the experimental room $24 \mathrm{~h}$ before behavioural testing for acclimatization. The animals were fed a standard laboratory diet and tap water ad libitum and kept at $23{ }^{\circ} \mathrm{C}$ with a $12 \mathrm{~h} \mathrm{light/dark} \mathrm{cycle,} \mathrm{light} \mathrm{on} \mathrm{at} \mathrm{07:00} \mathrm{h.} \mathrm{All} \mathrm{animal} \mathrm{care} \mathrm{and} \mathrm{ex-}$ perimental protocols were in compliance with international laws and policies (Directive 2010/63/EU of the European parliament and of the council of 22 September 2010 on the protection of animals used for scientific purposes; Guide for the Care and Use of Laboratory Animals, US National Research Council, 2011) and were approved by the Animal Care and Research Ethics Committee of the University of Florence, Italy, under license from the Italian Department of Health (54/2014-B, 410/2017-PR). Animal studies are reported in compliance with the animal research: reporting of in vivo experiments (ARRIVE) guidelines (Kilkenny et al., 2010; McGrath and Lilley, 2015). All efforts was taken to minimize the number of animals used and their suffering. Mice were sacrificed by cervical dislocation for removal of spinal cord for in vitro analysis. The number of animals per experiment was based on a power analysis (Charan and Kantharia, 2013) and calculated by G power software. To determine the anti-nociceptive effect, each tested group comprised 8 animals.

\section{Drug administration}

Mice were randomly assigned to each group by an individual other than the operator. ZOE was dissolved in $1 \%$ sodium carboxymethyl cellulose (CMC) and administered by gavage before testing at the doses of 100,200 and $400 \mathrm{mg} \mathrm{kg}^{-1}$ to determine the dose-response curve. For repeated administration, ZOE $\left(200 \mathrm{mg} \mathrm{kg}^{-1}\right)$ was administered once daily by gavage for 7 days starting from three days after surgery. The control group received equivalent volume of the vehicle.

Pregabalin (30 mg kg $\mathrm{mg}^{-1}$ i.p) (Sigma Aldrich) used as a reference drug, was dissolved in saline and administered intraperitoneally $3 \mathrm{~h}$ before testing. ( \pm )-baclofen (BACL) $\left(4 \mathrm{mg} \mathrm{kg}^{-1} \mathrm{s.c}\right.$ ) (Sigma Aldirch) used as a reference drug was dissolved in saline and administered with subcutaneous injection 45 min before testing. D-amphetamine hydrochloride (AMPH) $\left(2 \mathrm{mg} \mathrm{kg}^{-1}\right.$ i.p) (Sigma Aldrich) used as a reference drug was dissolved in saline and administered intreaperitoneally 15 min before testing.

LG325, a selective histone deacetylase 1 (HDAC1) inhibitor, was synthesized in the laboratory of Prof. Maria Novella Romanelli (University of Florence, Italy) and administered $5 \mu \mathrm{g}$ per mouse by intrathecal (i.t.) injection 15 min before the tests, as previously described (Sanna et al., 2017). U0126, a well-known mitogen-activated protein kinase (MEK) inhibitor, (20 $\mu \mathrm{g}$ per mouse) was dissolved in $20 \%$ dimethyl sulfoxide (DMSO) and it was administered i.t. $60 \mathrm{~min}$ before testing.

\section{SNI procedure}

Behavioural tests were performed before surgery to establish a baseline for comparison with postsurgical values. Surgery was performed as described by others (Bourquin et al., 2006). Mice were anesthetized with a mixture of $4 \%$ isoflurane in $\mathrm{O}_{2} / \mathrm{N}_{2} \mathrm{O}(30: 70 \mathrm{v} / \mathrm{v})$ and placed in a prone position. The right hind limb was slightly elevated and a skin incision was made on the lateral surface of the thigh. The sciatic nerve was exposed at mid-thigh level distal to the trifurcation and freed of connective tissue; the three peripheral branches (sural, common peroneal and tibial nerves) of the sciatic nerve were exposed without hurting nerve structures. Both tibial and common peroneal nerves were ligated with a microsurgical forceps (5.0 silk, Ethicon; Johnson \& Johnson Intl, Brussels, Belgium) and transacted together. The sural nerve was carefully preserved by avoiding any nerve stretch or contact with surgical tools. Muscle and skin were closed in two distinct layers with silk 5.0 sutures. Intense, reproducible and longlasting thermal and mechanical allodynia-like behaviour are measurable in the non-injured sural nerve skin extensions. The sham procedure consisted of the same surgery without ligation and transection of the nerves.

\section{Nociceptive behaviour}

Animals were habituated to the testing environment daily for at least 2 days before baseline testing. Nociceptive responses to a mechanical or thermal stimulus were measured before and 3, 7 and 10 days after surgery and each mouse possess its own control. All testing was performed with a blind procedure.

\section{Mechanical threshold (von Frey's test)}

Mechanical allodynia was measured by using a Dynamic Plantar Aesthesiometer (Ugo Basile, Gemonio, Italy) as previously described (Sanna et al., 2015).

\section{Hargreaves' plantar test}

The thermal nociceptive threshold was measured using Hargreaves' device (Hargreaves et al., 1988). Paw withdrawal latency in response to infrared heat was assessed using the plantar test apparatus (Ugo Basile). Each mouse was placed under a transparent plexiglass box $(7.0 \times 12.5$ $\mathrm{cm}^{2}, 17.0 \mathrm{~cm}$ high) on a 0.6 -cm-thick glass plate and allowed to acclimatize for $1 \mathrm{~h}$ before recording. The radiant heat source consisted of an infrared bulb (Osram halogen-bellaphot bulb; $8 \mathrm{~V}, 50 \mathrm{~W}$ ) that was positioned $0.5 \mathrm{~cm}$ under the glass plate directly beneath the hind paw. The time elapsed between switching on the infrared radiant heat stimulus and paw withdrawal response was measured automatically. The intensity of the infrared light beam was chosen to give baseline latencies of $10 \mathrm{~s}$ in control mice. A cut-off of $20 \mathrm{~s}$ was used to prevent tissue damage. Each hind paw was tested two-three times, alternating between paws with an interval of at least 1 min between tests. The interval between two trials on the same paw was of at least $5 \mathrm{~min}$. Nociceptive response for thermal sensitivity was expressed as thermal paw withdrawal latency in seconds. All determinations were averaged for each animal.

\section{Locomotor activity}

\section{Rotarod test}

Possible side effects of ZOE on motor performance were assessed by rotarod test, as previously described (Sanna et al., 2019).

\section{Hole-board test}

The spontaneous locomotor activity was evaluated by using the hole-board test, as previously described (Sanna et al., 2019).

\section{In vitro neuroinflammation and neuroprotective model}

For detailed description of the cell culture conditions and the cell viability assay please see Supplementary information. In brief, immortalised murine microglial cells (BV2) were seeded in 6-well plates $\left(3 \times 10^{5}\right.$ cells/well) and pre-treated with ZOE $10 \mu \mathrm{g} / \mathrm{ml}$ or GIN, SHO and ZTE at the respective concentrations in the extract, for $4 \mathrm{~h}$ and then stimulated with LPS $250 \mathrm{ng} / \mathrm{ml}$ for $24 \mathrm{~h}$. Then, the conditioned BV2 
medium was collected and centrifuged $\left(1000 \mathrm{x} \mathrm{g}\right.$ for $\left.10 \mathrm{~min}, 37^{\circ} \mathrm{C}\right)$. The pellet was discarded and the supernatant was stored at $-80^{\circ} \mathrm{C}$ for cytokines dosages or used to treat immortalised human neuroblastoma (SH-SY5Y) cells $\left(5 \times 10^{5}\right.$ cells/well in $96-$ well plates). The neuroprotective effect of ZOE and its constituents was evaluated by assessing the cell viability of the SH-SY5Y cells treated with the conditioned BV2 medium for $24 \mathrm{~h}$. Unstimulated BV2 medium was used as control. LG325 $(5 \mu \mathrm{M})$ and U0126 $(5 \mu \mathrm{M})$ were used as HDAC1 and MEK positive control, respectively.

Preparation of tissue and cell lysates, ELISA, western blot and immunofluorescence

The detailed protocols used for preparing tissue and cell lysates and for performing enzyme-linked immunoabsorbant assay (ELISA), western blot and immunofluorescence are described in the Supplementary information.

\section{Statistical analysis}

The data and statistical analysis in this study comply with the recommendation on experimental design and analysis in pharmacology (Curtis et al., 2018). The behavioural data are presented as means \pm SEM. Eight mice per group were used. Two-way analysis of variance (ANOVA) followed by Bonferroni post hoc was used for statistical analysis. For the locomotor activity, the unpaired sample $t$-test was performed. For in vitro analysis data are expressed as the mean \pm SEM of five experiments and assessed by One-way ANOVA followed by Tukey post hoc test. For each test a value of $p<0.05$ was considered significant. The computer programme GraphPad Prism version 5.0 (GraphPad Software, San Diego, CA, USA) was used in all statistical analysis.

\section{Results}

\section{Chemical characterization of ZOE}

The chemical characterization of ZOE is shown in the Tables 1 and 2 and the HPLC and GC chromatograms are reported in the Supplementary information (Fig. 1S and 2S). ZOE contains $24.73 \%$ total gingerols and $3.03 \%$ total shogaols, and the amount of GIN and SHO resulted to be $10.07 \%$ and $1.68 \%$, respectively.

Regarding the volatile fraction, ZTE was found to be $30.10 \%$, with zingiberene being the most abundant volatile constituent (28.2\%).

\section{Effect of ZOE on anti-nociceptive activity}

SNI induced mechanical (Fig. 1A) and thermal allodynia (Fig. 1D) in the ipsilateral side, starting from 3 days after surgery up to day 21 . No hypersensitivity was observed in the contralateral side (Fig. 1A, D). The dose-response study performed seven days after surgery showed that oral administration of ZOE completely prevented mechanical (Fig. 1B) and thermal allodynia (Fig. 1E) at the dose of $200 \mathrm{mg} \mathrm{kg}^{-1}$ and no further increase of activity was observed at higher doses. The dose of $100 \mathrm{mg} \mathrm{kg}^{-1}$ was ineffective. Time-course experiments showed that, 30 min after the administration, ZOE $200 \mathrm{mg} \mathrm{kg}^{-1}$ has a tendency to

Table 1

HPLC-DAD quantification of 6-gingerol (GIN), 6shogaol (SHO) and terpenoid-enriched fraction (ZTE) in the standardized extract from Zingiber officinale Roscoe rhizomes (ZOE). Data are expressed as $\% \pm$ standard deviation

\begin{tabular}{ll}
\hline Compound & $\%$ \\
\hline GIN & $10.07 \pm 0.51$ \\
SHO & $1.68 \pm 0.33$ \\
ZTE & $30.10 \pm 1.50$ \\
\hline
\end{tabular}

Table 2

Chemical composition of the volatile compounds in the standardized extract from Zingiber officinale Roscoe rhizomes (ZOE). Data are expressed as \% relative peak area \pm standard deviation

\begin{tabular}{|c|c|c|c|}
\hline Peak number & Compound & $L R I$ & $\%$ Area \\
\hline 1 & Ethyl butanoate & 805 & $1.7 \pm 0.9$ \\
\hline 2 & $\alpha$-pinene & 933 & $1.5 \pm 0.2$ \\
\hline 3 & Camphene & 947 & $4.1 \pm 0.8$ \\
\hline 4 & $\beta$-pinene & 976 & $0.2^{\mathrm{a}}$ \\
\hline 5 & $\beta$-myrcene & 988 & $0.3^{\mathrm{a}}$ \\
\hline 6 & Octanal & 992 & $0.8 \pm 0.1$ \\
\hline 7 & $\alpha$-phellandrene & 1005 & $0.7 \pm 0.2$ \\
\hline 8 & $\beta$-thujene & 1029 & $4.6 \pm 1.1$ \\
\hline 9 & 1,8-cineole & 1031 & $2.3 \pm 0.4$ \\
\hline 10 & Terpinolene & 1088 & $0.3^{\mathrm{a}}$ \\
\hline 11 & $\beta$-linalool & 1101 & $0.9^{\mathrm{a}}$ \\
\hline 12 & Endoborneol & 1166 & $1.4 \pm 0.1$ \\
\hline 13 & Terpinen-4-ol & 1178 & $0.3^{\mathrm{a}}$ \\
\hline 14 & $\alpha$-terpineol & 1191 & $0.8 \pm 0.3$ \\
\hline 15 & Verbenone & 1208 & $0.7 \pm 0.5$ \\
\hline 16 & Neral & 1246 & $0.9^{\mathrm{a}}$ \\
\hline 17 & Geraniol & 1260 & $0.4 \pm 0.2$ \\
\hline 18 & Geranial & 1275 & $1.5^{\mathrm{a}}$ \\
\hline 19 & $\alpha$-copaene & 1380 & $0.6 \pm 0.2$ \\
\hline 20 & $\beta$-elemene & 1386 & $0.6^{\mathrm{a}}$ \\
\hline 21 & $\alpha$-cedrene & 1395 & $0.8 \pm 0.3$ \\
\hline 22 & $\alpha$-gurjunene & 1409 & $0.6 \pm 0.3$ \\
\hline 23 & $\alpha$-bergamotene & 1439 & $0.7 \pm 0.2$ \\
\hline 24 & $\beta$-farnesene & 1461 & $0.8 \pm 0.1$ \\
\hline 25 & Aromadendrene & 1468 & $0.5 \pm 0.2$ \\
\hline 26 & Germacrene D & 1484 & $0.6 \pm 0.2$ \\
\hline 27 & $\alpha$-curcumene & 1488 & $6.2 \pm 0.1$ \\
\hline 28 & Zingiberene & 1502 & $28.2 \pm 0.3$ \\
\hline 29 & $\gamma$-muurolene & 1505 & $2.3 \pm 0.2$ \\
\hline 30 & $(\mathrm{E}, \mathrm{E})$ - $\alpha$-farnesene & 1513 & $6.9^{\mathrm{a}}$ \\
\hline 31 & $\beta$-bisabolene & 1515 & $4.3^{\mathrm{a}}$ \\
\hline 32 & $\beta$-sesquiphellandrene & 1531 & $11.0 \pm 0.2$ \\
\hline 33 & $\delta$-cadinene & 1538 & $0.5^{\mathrm{a}}$ \\
\hline 34 & (E)-nerolidol & 1560 & $0.1^{\mathrm{a}}$ \\
\hline \multirow[t]{2}{*}{35} & Germacrene B & 1568 & $0.4 \pm 0.1$ \\
\hline & TOTAL & & $88.6 \pm 0.8$ \\
\hline
\end{tabular}

${ }^{\text {a }} \mathrm{SD}<0.05$

reduce nociceptive symptoms, which became statistically significant after $60 \mathrm{~min}$ and peaked at $120 \mathrm{~min}$ (Fig. 1B, E). Although it did not reach significant, ZOE still maintains a tendency to reduce allodynia three $\mathrm{h}$ after administration. Notably, the intensity of the anti-hyperalgesic effect of ZOE $200 \mathrm{mg} \mathrm{kg}^{-1}$ was similar to that induced by pregabalin (30 $\mathrm{mg} \mathrm{kg}^{-1}$ ), used as positive control, without any effect on contralateral side (Fig. 1E, F).

Oral repeated administration of ZOE $200 \mathrm{mg} \mathrm{kg}^{-1}$ reverted mechanical allodynia in the ipsilateral side 7 and 10 days post-surgery (Fig. 1G). The administration of ZOE on SNI exposed to repeated administration of the extract showed a time course similar to the acute administration, with a maximum effect between 60 and $120 \mathrm{~min}$ (Fig. 1H). Repeated exposure to ZOE induced a significant increase of the basal mechanical threshold compared to untreated SNI mice. ZOE administration in mice exposed to repeated treatment induced a peak effect more consistent than acute administration. Repeated administration showed a trend (that did not reach significance) towards an increase in the contralateral side thermal and mechanical threshold (Fig. 1I).

\section{Effect of ZOE p.o. administration on locomotor activity}

Mice treated with the highest effective dose of ZOE (200 $\mathrm{mg} \mathrm{kg}^{-1}$ ) were evaluated for motor coordination, by means of the rotarod test, and for spontaneous mobility and exploratory activity, by means of the hole board test. Overall, ZOE-exposed SNI mice did not show any gross behaviour alterations. Oral administration of ZOE did not alter motor 

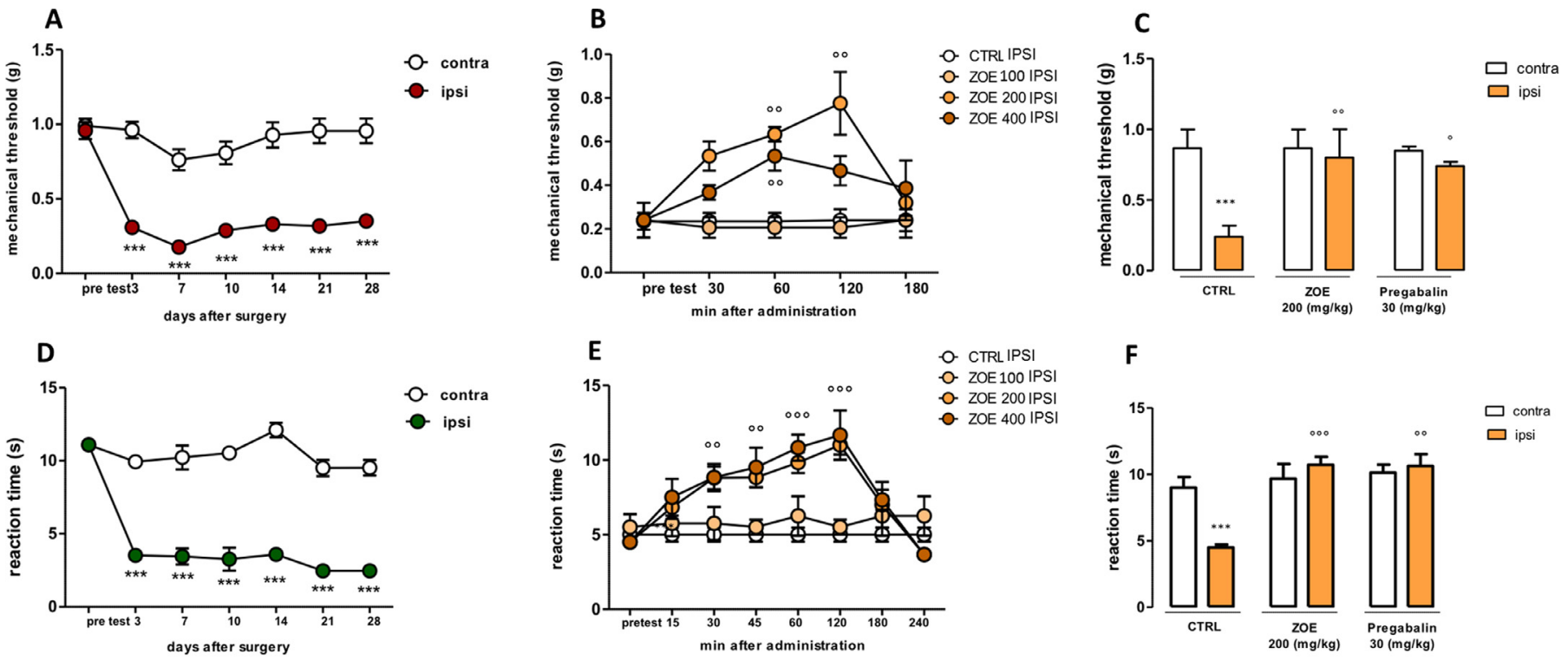

F

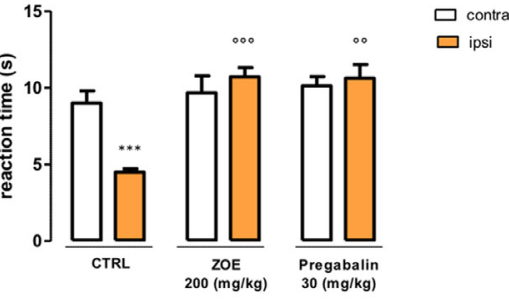

G

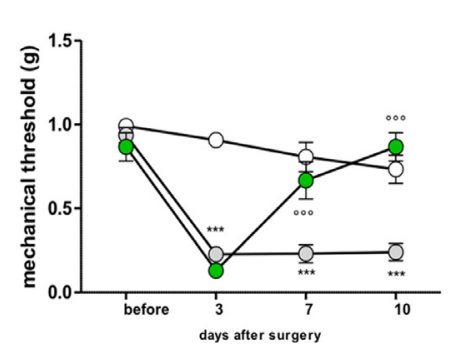

H

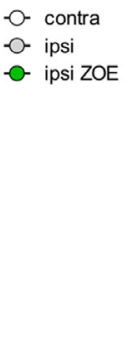

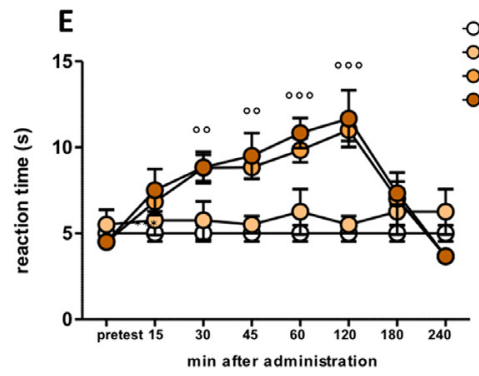

- CTRLIPSI

O. ZOE 100 IPSI

O. ZOE 200 IPSI
- ZOE 400 IPSI

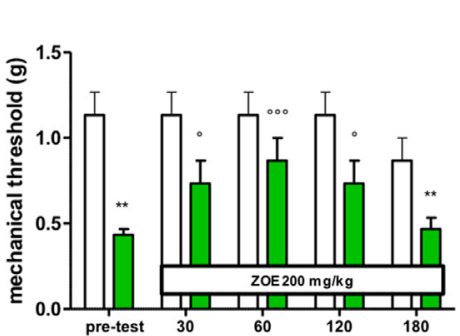

I

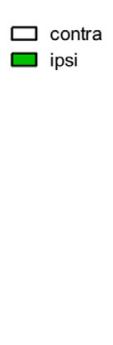

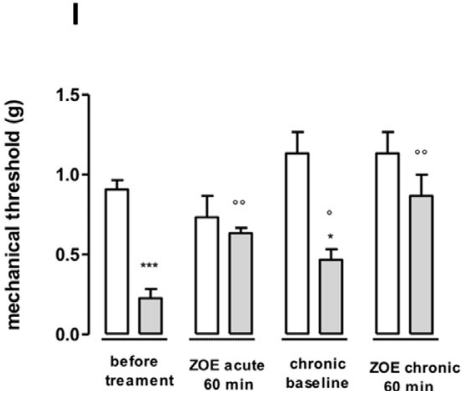

Fig. 1. Anti-nociceptive effects of the standardized extract from Zingiber officinale Roscoe rhizomes (ZOE) in SNI mice. (A) The long-lasting mechanical allodynia produced by SNI up to 21 days after surgery. (B) The dose-response and the time course curve showed the effect of ZOE 100,200 and $400 \mathrm{mg} \mathrm{kg}^{-1}$ against mechanical allodynia. (C) Comparison of the effect of ZOE (p.o. $200 \mathrm{mg} \mathrm{kg}^{-1}$ ) on mechanical allodynia to that produced by pregabalin (i.p $30 \mathrm{mg}$ kg ${ }^{-1}$ ). (D) The long-lasting thermal hyperalgesia produced by SNI model in mice. (E) The effect of acute oral administration of ZOE 100,200 and $400 \mathrm{mg} \mathrm{kg}^{-1}$ on thermal allodynia after SNI surgery. (F) Comparison of the effect of ZOE $200 \mathrm{mg} \mathrm{kg}-1$ on thermal hyperalgesia to that produced by pregabalin (i.p $30 \mathrm{mg} \mathrm{kg}^{-1}$ ). (G) The effect of ZOE $200 \mathrm{mg} / \mathrm{kg}$ on mechanical allodynia at 3, 7 and 10 days after surgery. (H) Time course of oral repeated administration of ZOE $200 \mathrm{mg} \mathrm{kg}^{-1}$. (I) Comparison of the effect on mechanical allodynia between acute and repeated administration of ZOE $200 \mathrm{mg} \mathrm{kg}^{-1}$. Data points represent the mean \pm SEM obtained from experiments conducted on 8 mice. ${ }^{* * *} p<0.001 ;{ }^{* *} p<0.01 ;{ }^{*} p<0.05 v$ s. contralateral side; ${ }^{\circ 00} p<0.001 ;{ }^{\circ} p<0.01 ;{ }^{\circ} p<0.05 v s$. ipsilateral side before the treatment.

coordination (Fig. 2A), exploratory activity (Fig. 2B) or spontaneous mobility (Fig. 2C), compared to the control group.

\section{Effect of ZOE on ERK 1/2 activation in vivo and in vitro}

Mitogen-activated protein kinases (MAPKs) play an important role in the spinal mechanism of NP and neuroinflammation (Edelmayer et al., 2014). The role of these kinases in the cellular mechanism of action of ZOE was, thus, investigated in SNI animals and in BV2 cells. Consistently with other published work (Subedi et al., 2019), the peak of extracellular signal-regulated kinase (ERK) and p38 mitogen-activated protein kinases (p38) MAPKs phosphorylation in BV2 cells was observed at $30 \mathrm{~min}$ of LPS stimulation. The reliability of the in vitro model was confirmed by using U0126 $10 \mu \mathrm{M}$, a well-known MEK inhibitor, as a positive control. ZOE was used at the concentration of 10 $\mu \mathrm{g} / \mathrm{ml}$ according to previously performed cell viability assay (Fig. 3S; Supplementary information) and it was able to reduce ERK1/2 activation (Fig. 3A; 3B), while no effect was observed on p38 phosphorylation (Fig. 3C). To confirm that the effect observed in ZOE-treated BV2 cell concurs with the in vivo mechanism of action, we evaluated its effects
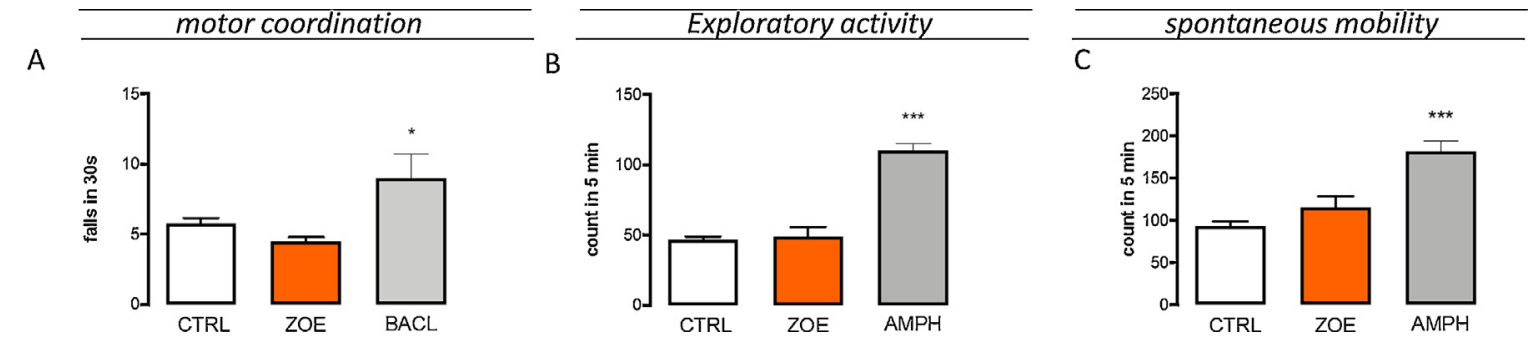

Fig. 2. Lack of effect of the standardized extract from Zingiber officinale Roscoe rhizomes (ZOE) on locomotor behaviour. Lack of impairment of (A) motor coordination compared to BACL (* $p<0.05 v s$. CTRL), (B) exploratory activity and (C) spontaneous mobility compared to AMPH (***p $p<0.001 v s$. CTRL) in mice treated with ZOE $200 \mathrm{mg} \mathrm{kg}^{-1}$. Data were recorded at the peak of antinociceptive activity. 


\section{BV2}

A

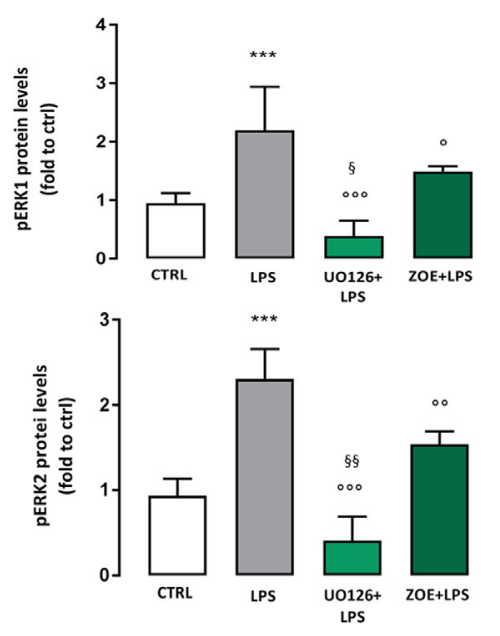

C
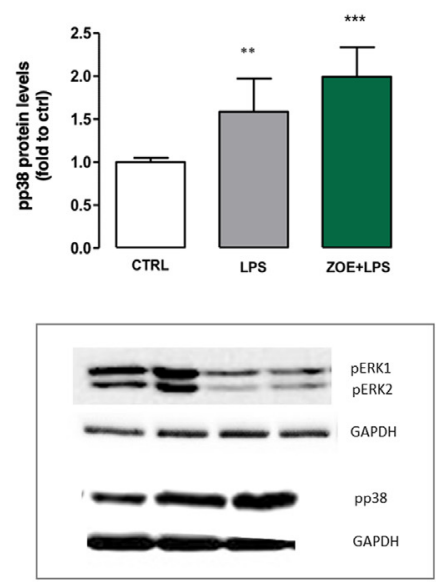

SNI ACUTE ADMINISTRATION

D
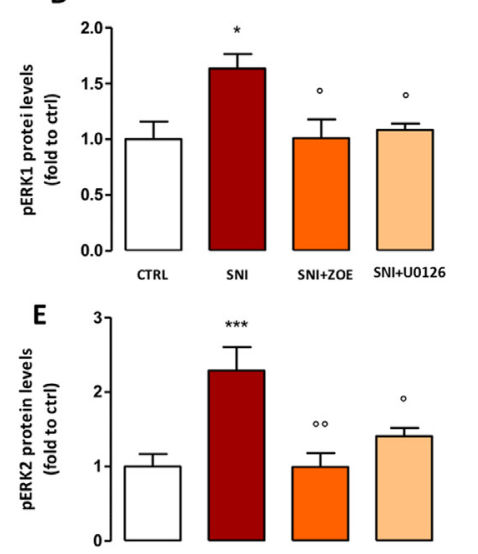

CTRL

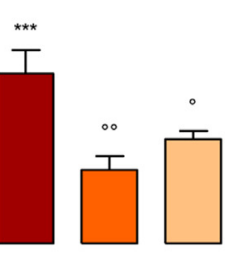

SNI

SNI+ZOE $\quad$ SNI+U0126

H

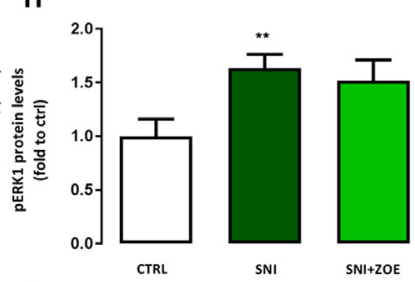

I

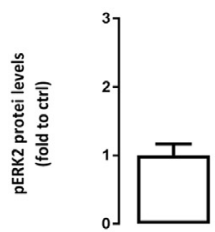

CTRL

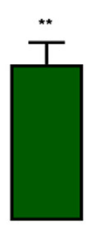

SNI

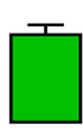

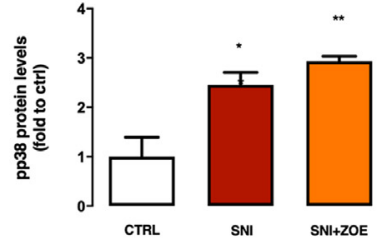

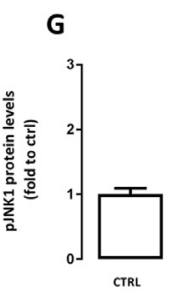

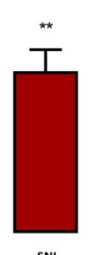

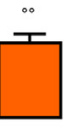

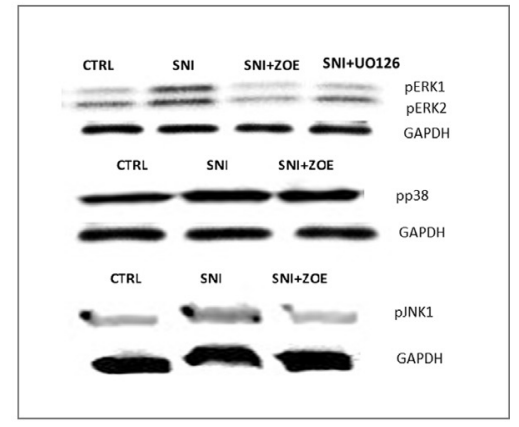

\section{SNI REPEATED ADMINISTRATION}

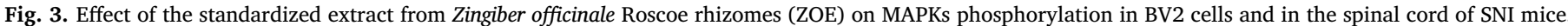

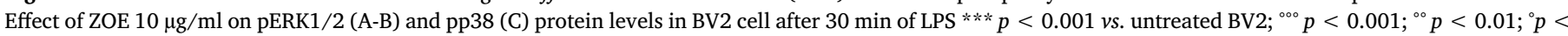

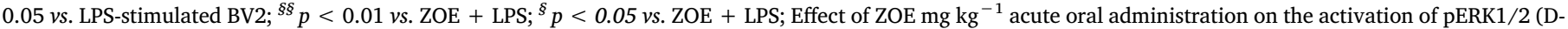

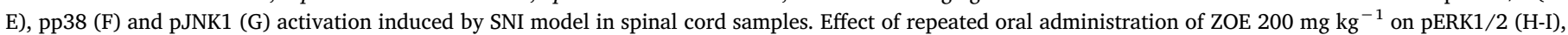

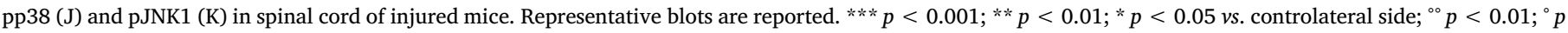
$<0.05$ vs. SNI mice. 

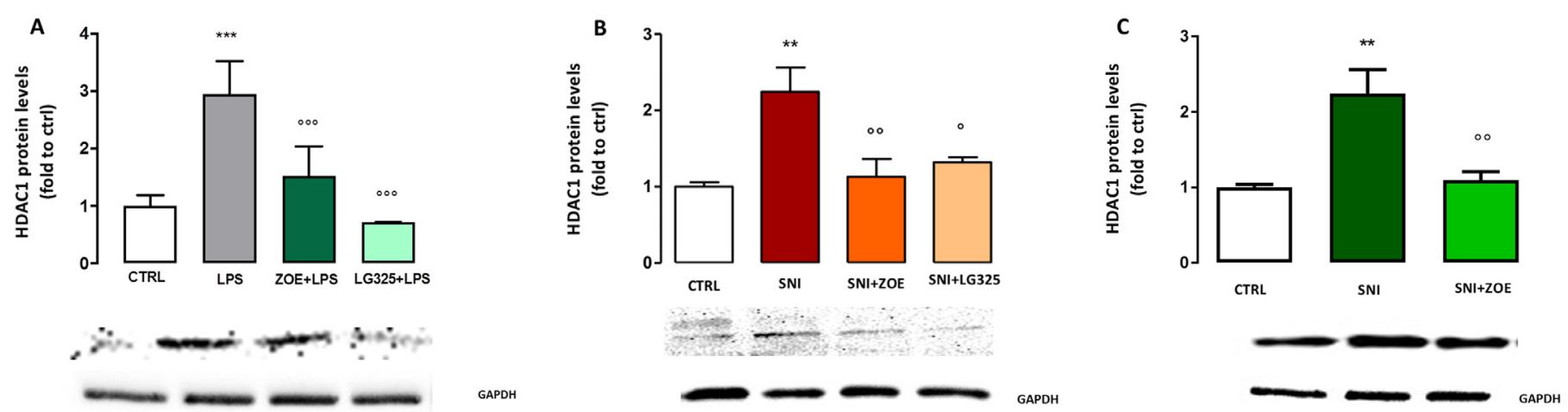

Fig. 4. Effect of the standardized extract from Zingiber officinale Roscoe rhizomes (ZOE) on HDAC1 protein expression. Effect of ZOE (10 $\mu \mathrm{g} / \mathrm{ml})$ on the HDAC1 expression in BV2 after $24 \mathrm{~h}$ of LPS stimulation (A). ${ }^{* * *} p<0.001 v s$. untreated BV2; ${ }^{\circ 0} p<0.001 v s$. LPS-stimulated BV2. Acute (B) and repeated (C) oral administration of ZOE $200 \mathrm{mg} \mathrm{kg}^{-1}$ effect on HDAC1 protein expression in SNI mice. Representative blots are reported. ${ }^{* *} p<0.01 ;{ }^{*} p<0.05 v s$. contralateral side; ${ }^{\circ} p<0.01 ;{ }^{\circ} p<0.05 v s$. SNI mice.

on MAPKs activation in SNI spinal cord mice samples.

An increase of phosphorylated ERK (pERK)1 (Fig. 3D, H) and pERK2 (Fig. 3E, I) was observed in the ipsilateral side of SNI mice. ZOE completely prevented ERK1 (Fig. 3D) and ERK2 (Fig. 3E) activation after acute administration, similarly to U0126 used as positive control. pERK2 levels were also reduced after repeated treatment (Fig. 3I), whereas no effect on ERK1 phosphorylation was observed (Fig. 3H). An increase of phosphorylated p-38 (pp38) levels was observed in the spinal cord of SNI mice. Consistently with the results obtained in BV2 cells, ZOE did not reduce p38 phosphorylation, neither after acute (Fig. 3F) nor repeated administration (Fig. 3J).

In agreement with the literature (Zhuang et al., 2006), SNI surgery group showed an isoform-selective activation for c-Jun n-terminal kinase (JNK), with phosphorylated JNK1 (pJNK1) (p46) being altered in the injured spinal cord. Acute and repeated treatment with ZOE showed a tendency to reduce pJNK1 activation (Fig. 3G, K), compared to pERK1.

Effect of ZOE on HDAC1 protein expression in the spinal cord

As shown in Fig. 4A, LPS induced the up-regulation of HDAC1 protein expression in BV2 cells. This effect was completely prevented by ZOE pre-treatment, with a similar trend of LG325 a selective HDAC1 inhibitor (Sanna et al.,2017).

Consistently with our previous study (Sanna et al., 2017), SNI mice showed an increased expression of HDAC1 protein in the ipsilateral side compared to contralateral side. The increase of HDAC1 was significantly prevented by acute (Fig. 4B) and repeated (Fig. 4C) ZOE administration, with the efficacy being comparable to LG325.

Effect of ZOE on NF-кBp65 nuclear translocation in SNI mice and in BV2 cells

Nuclear factor kappa-light-chain enhancer of activated B cells subunit p65 (NF-kBp65) is one of the most important pathways involved in inflammatory processes (Shih et al., 2015). Thus, here we investigated its role in ZOE mechanism of action.

Time-course studies showed that LPS stimulation induced a phosphorylated-p65 (pp65) nuclear translocation that peaked $1 \mathrm{~h}$ after treatment (Fig. 4S; Supplementary information). To evaluate the effect of ZOE on pp65 nuclear translocation, immunofluorescence experiments were carried out. We found pp65 subunit primarily present in the cytoplasm in unstimulated cells (Fig. 5A), whereas, pp65 level in the nucleus were increased after $1 \mathrm{~h}$ of LPS stimulation (Fig. 5B). The treatment with ZOE $10 \mu \mathrm{g} / \mathrm{ml}$ blocked the nuclear translocation in LPSstimulated cells (Fig. 5C). Analysis by fluorescent staining intensity confirmed these results. In fact, the intensity of nuclear fluorescence of pp65 in LPS stimulated cells resulted significantly higher than in the control group and ZOE pre-treatment prevented pp65 nuclear translocation (Fig. 5D). To confirm that ZOE counteracted the activity of NFкBp65 we showed that $24 \mathrm{~h}$ after LPS stimulation the total levels of nuclear factor of kappa light polypeptide gene enhancer in B-cells inhibitor alpha (IKB $\alpha$ ) increased and ZOE treatment completely reverted this event (Fig. 5E).

In the spinal cord of SNI mice, we observed an increase of pp65 protein levels, which was completely reverted by ZOE acute treatment (Fig. 5F). A similar trend was observed for repeated administration, where the expression levels of pp65 were reduced compared to untreated mice (Fig. 5G).

LPS stimulation led to a significant release of the pro-inflammatory cytokines tumor necrosis factor- $\alpha$ (TNF- $\alpha$ ) (Fig. 6A), interleukin-6 (IL6) (Fig. 6B) and interleukin-1 $\beta$ (IL-1 $\beta$ ) (Fig. 6C) in BV2 culture medium, which was strongly prevented by ZOE pre-treatment. This effect is confirmed in ipsilateral side of SNI mice, where ZOE significantly reduced IL-1 $\beta$ expression levels after both acute (Fig. 5D) and repeated administration (Fig. 5E).

\section{Effects of ZOE main components in its biological activity}

To identify which constituent of ZOE was responsible for its biological effects, we tested the activity of GIN, SHO and ZTE, at the concentration present in the active dose of ZOE, on pERK activation, HDAC1 protein levels and NF-kBp65 pathway activation.

Similarly to ZOE, the pre-treatment of BV2 cells with GIN $(1 \mu \mathrm{g}$ $\left.\mathrm{ml}^{-1}\right)$ and SHO $\left(0.17 \mu \mathrm{g} \mathrm{ml}^{-1}\right)$ decreased LPS-induced ERK1/2 phosphorylation (Fig. 7A, B). In contrast, no effect was observed with ZTE (3 $\mu \mathrm{g} \mathrm{ml}^{-1}$ ) treatment. To dissect the role of MEK1 and the possible binding mode of ZOE constituents in its active site, we performed molecular docking simulations, using U0126 as a positive control. The docking software was able to accurately predict the binding mode of U0126, with a root-mean square deviation (RMSD) of 0.103 , compared to the original coordinates in the crystal structure. Gingerols and shogaols were able to bind the active site of MEK1 with binding energies ranging from $-7 \mathrm{kcal} / \mathrm{mol}$ to $-7.7 \mathrm{kcal} / \mathrm{mol}$, which are very similar to that obtained with the known inhibitors (Supplementary information), suggesting a similar affinity for the active site of MEK1. In contrast, the volatile compounds gave significantly higher binding energies, except for zingiberene, $\alpha$-copaene, $\alpha$-curcumene, $\beta$-bisabolene and $\beta$ - 
BV2

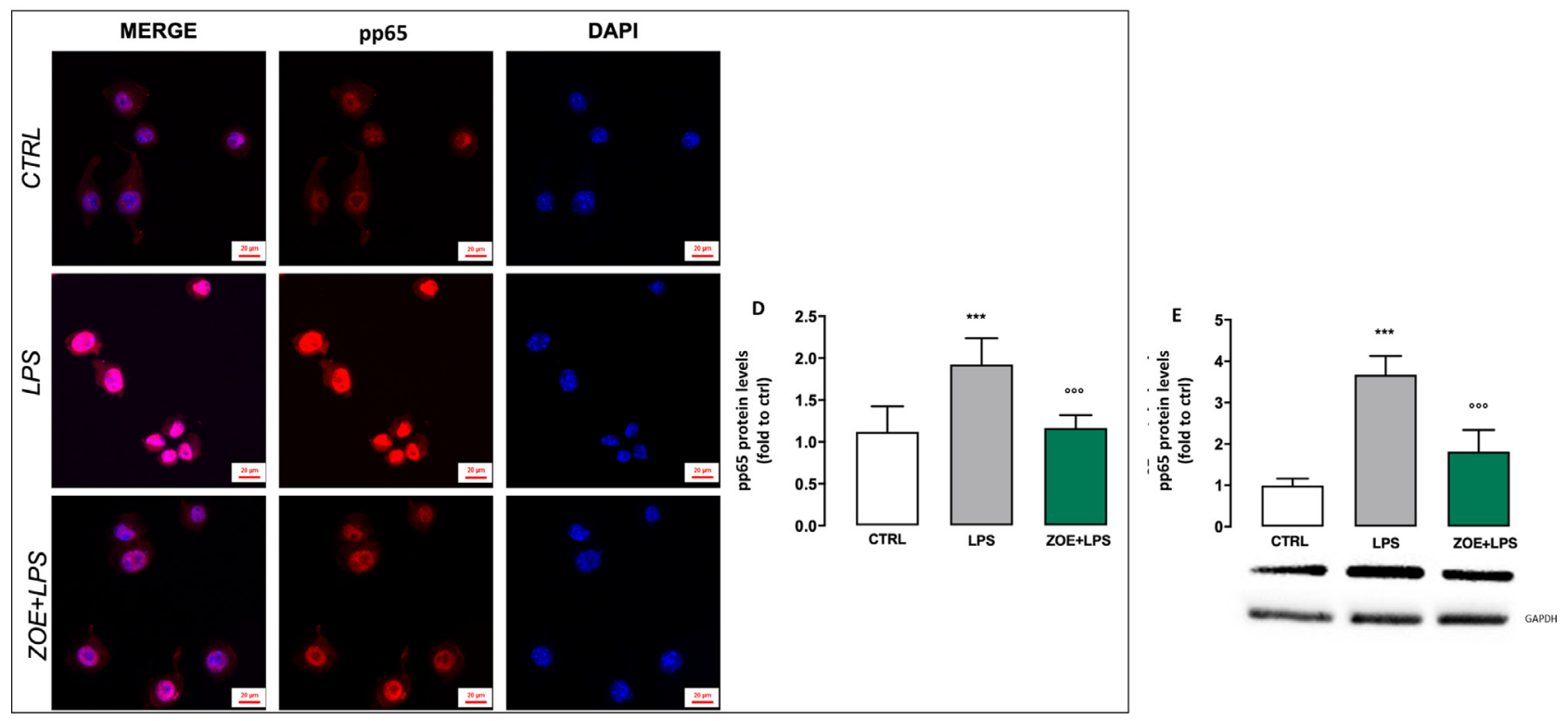

$$
\mathbf{F}
$$

SNI ACUTE ADMINISTRATION

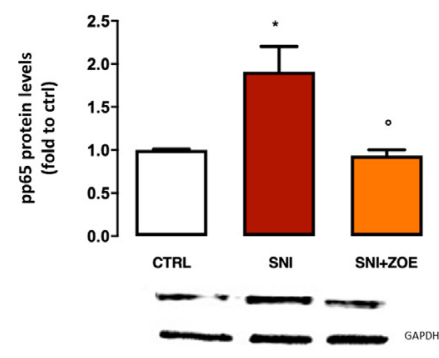

G

SNI REPEATED ADMINISTRATION

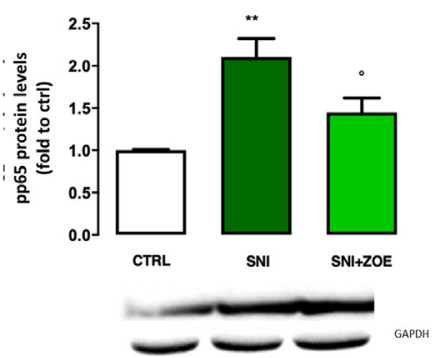

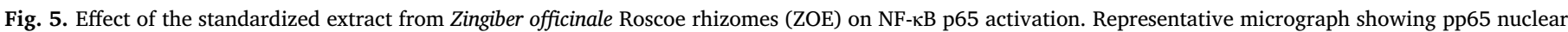

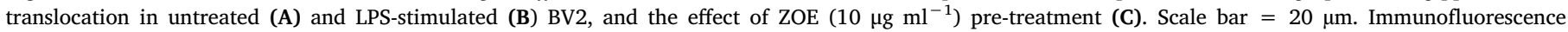

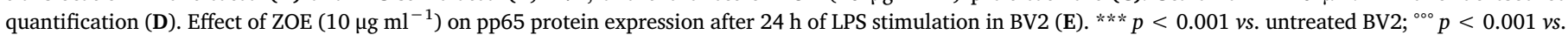

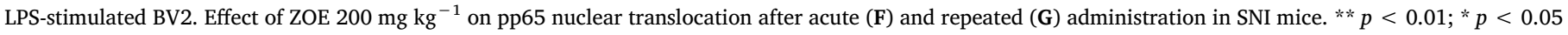
vs. controlateral side ${ }^{\circ} p<0.05 v s$. SNI mice).

sesquiphellandrene. However, the graphical analysis of the binding poses revealed that these volatile compounds were not able to replicate the key interactions needed for the inhibitor binding (Fig. 7C, D, E). Indeed, U0126 makes multiple hydrogen bonds involving the carbonyl group of ASP208, PHE209, and the amino group of LYS97, VAL211 and SER212. Similarly, the carbonyl moiety on the side chain of GIN and SHO is able to make hydrogen bonds with the amino group of VAL211 and SER212. Moreover, the hydroxyl substituent present on the side chain of GIN makes a hydrogen bond with the carbonyl group of PHE209. Both GIN and SHO completely occupy the cavity space, which accommodates U0126. In the contrast, the zingiberene hydrocarbon structure cannot make hydrogen bonds with the active site residues, suggesting a lower affinity for the MEK1 binding site.

Regarding HDAC1, ZTE showed an activity similar to ZOE leading to a strong reduction of protein expression. Conversely, GIN and SHO did not modulate this target (Fig. 7F).

Consistently, the increase of IKB $\alpha$ levels, observed after $24 \mathrm{~h}$ of LPS stimulation, was completely reverted by ZTE pre-treatment, with GIN and SHO showing no effect (Fig. 7G).

Inflammatory factors are known to induce neurotoxicity both in vivo and in vitro. Similarly to ZOE, ZTE pre-treatment significantly reduced the LPS-induced release of the pro-inflammatory cytokines TNF- $\alpha$ (Fig. 8A), IL-1ß (Fig. 8B) and IL-6 (Fig. 8C) by BV2 cells. In contrast, GIN and SHO did not show any significant effect.

\section{Effect of ZOE on inflammation-induced neurotoxicity in SH-SY5Y cells}

As shown in Fig. 8D, LPS-conditioned BV2 medium reduced SHSY5Y cell viability compared to the untreated control, suggesting that the pro-inflammatory cytokines secreted by LPS-activated microglia were able to induce neurotoxicity. LPS-conditioned medium obtained from ZOE-treated BV2 cells completely prevented this cytotoxic effect, returning to basal level. LPS-conditioned medium obtained from GIN-, SHO- and ZTE-treated BV2 cells was also able to significantly protect SH-SY5Y from the neurotoxic effect of BV2 conditioned medium.

\section{Discussion}

Currently, the available treatments for chronic NP are effective in fewer than $50 \%$ of patients, with a series of well-known side effects limiting the patients' compliance (Jensen and Finnerup, 2014). For this purpose, we investigated the pain relieving activity of ZOE in a mice model of peripheral neuropathy.

A single oral administration of ZOE completely reverted both mechanical and thermal allodynia in the ipsilateral side of SNI mice. Indeed, ZOE increased the pain threshold similarly to pregabalin, which is used as the first line treatment for NP. Despite there is convincing scientific evidence regarding the analgesic effect of ginger in the management of chronic inflammatory pain (Forouzanfar and Hosseinzadeh, 2018), to the best of our knowledge, no data are 
BV2

A

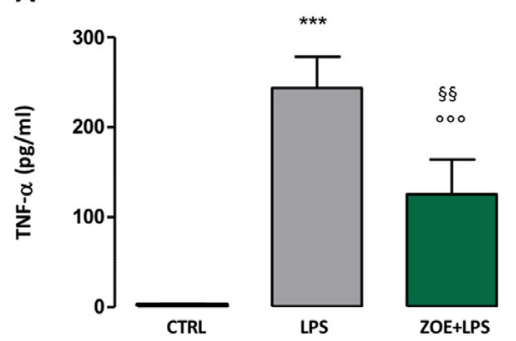

SNI ACUTE ADMINISTRATION

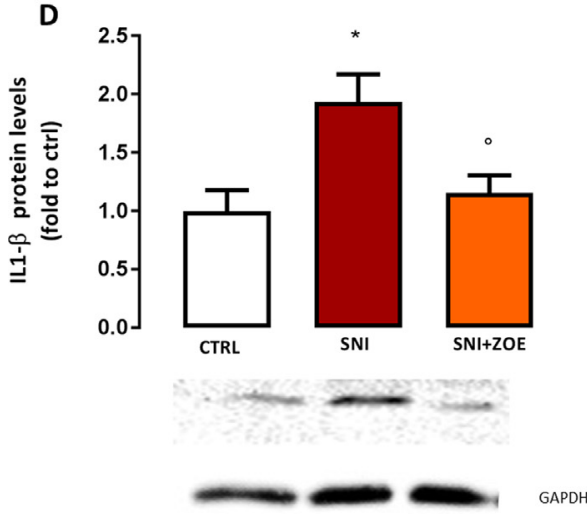

B

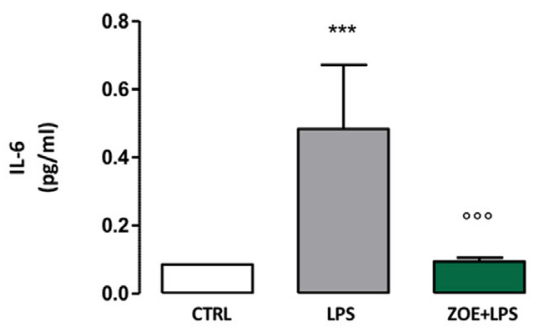

C

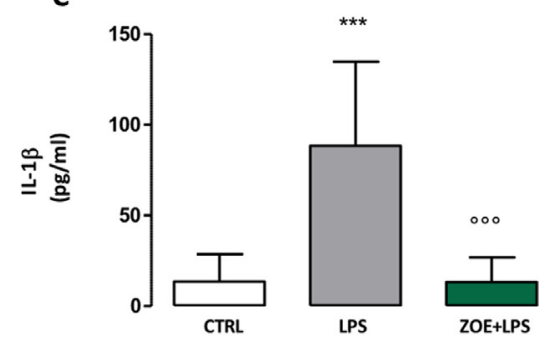

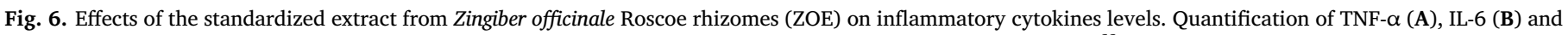

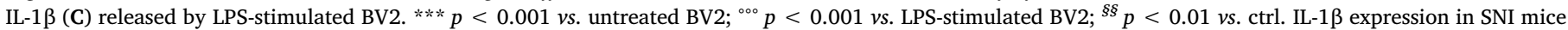

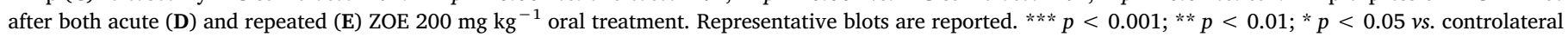
side; ${ }^{\circ} p<0.01 ;{ }^{\circ} p<0.05$ vs. SNI mice.

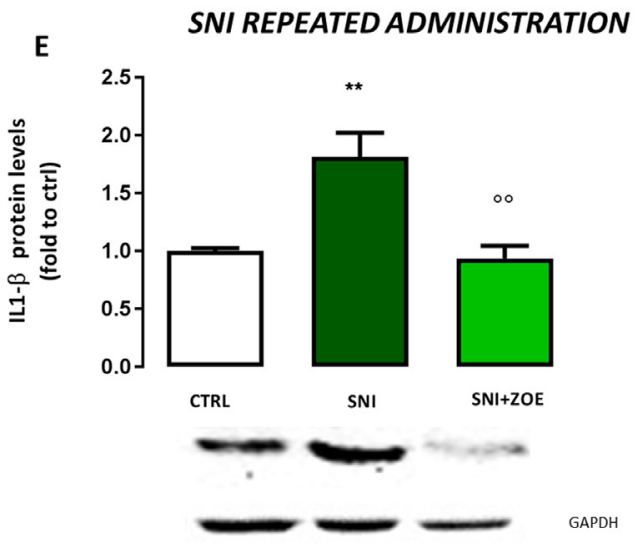

A

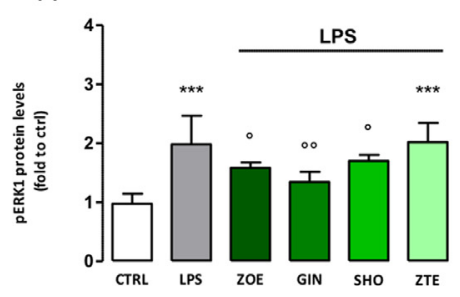

$\mathrm{F}$

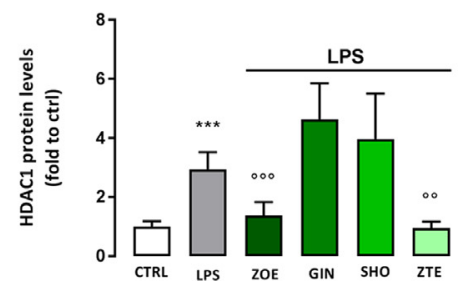

B
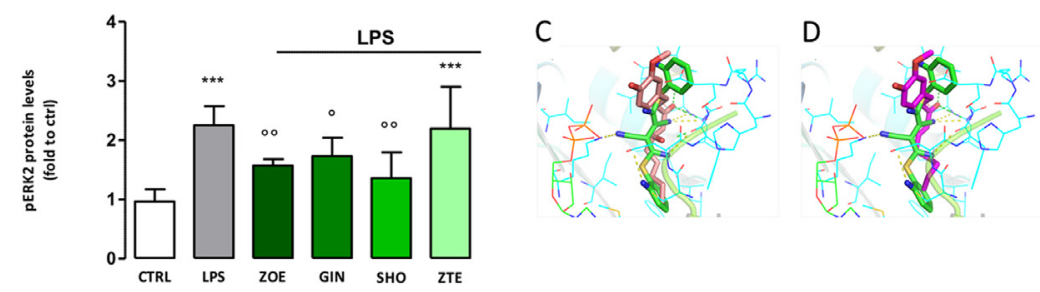

E

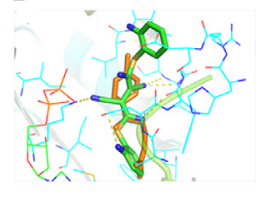

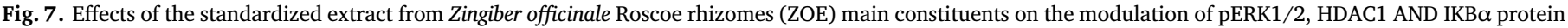

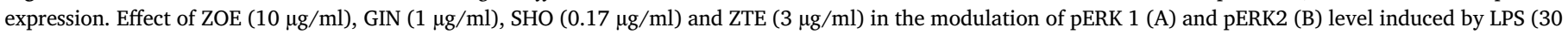

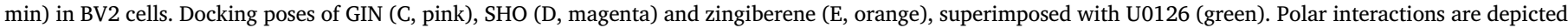

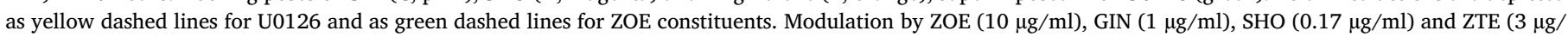

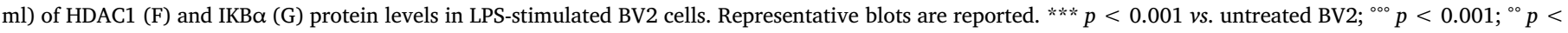
$0.01 ;{ }^{\circ} p<0.05 v$ s. LPS-stimulated BV2. 
A

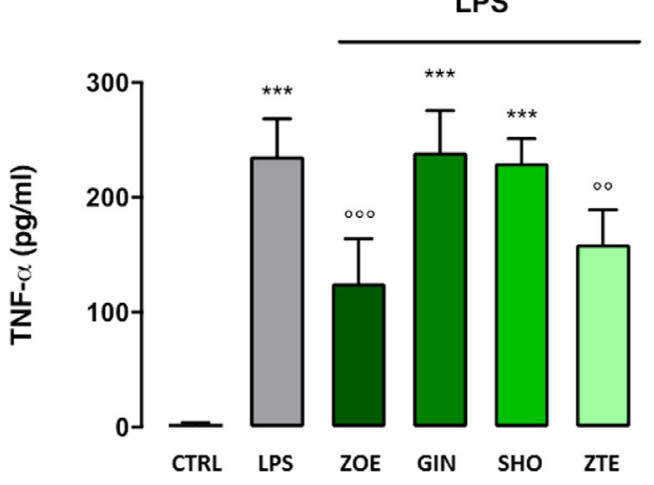

B

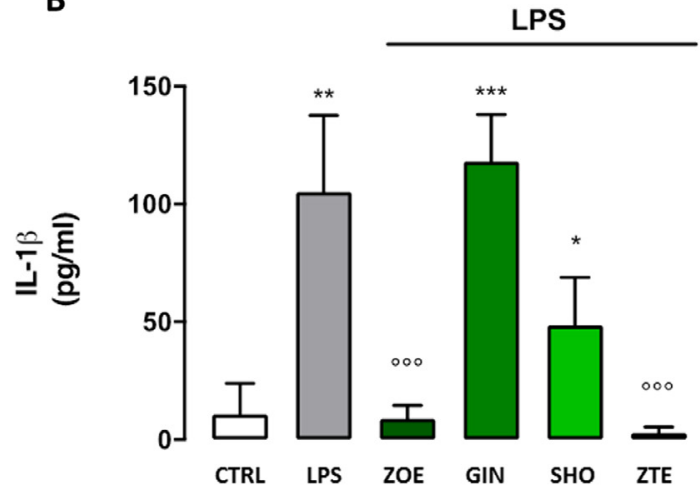

C

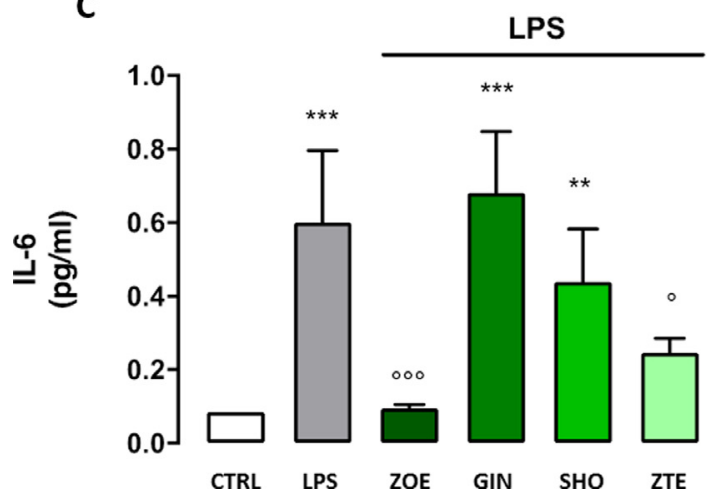

D

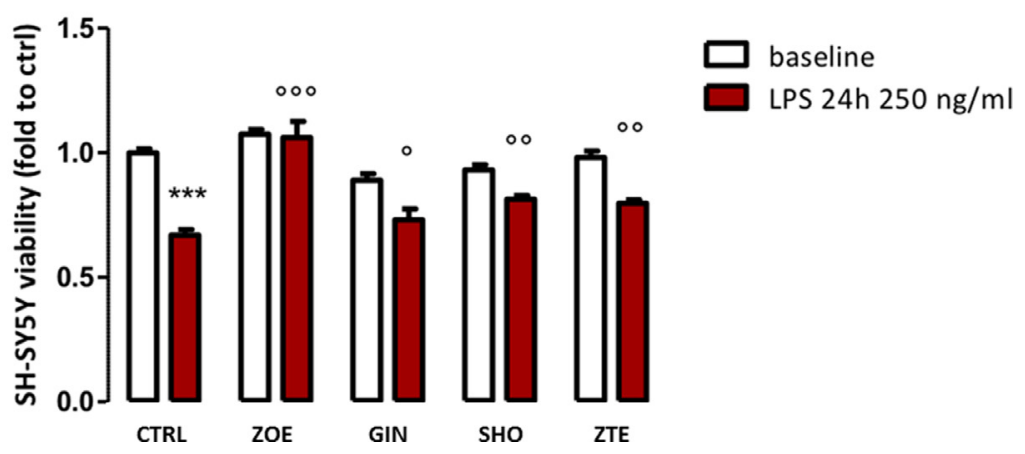

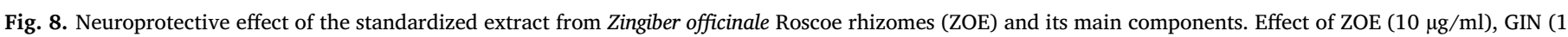

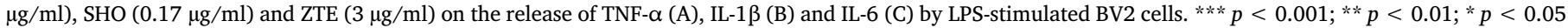

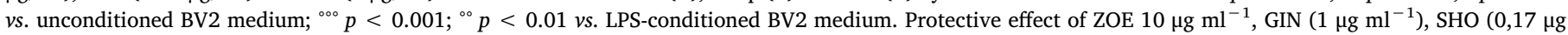

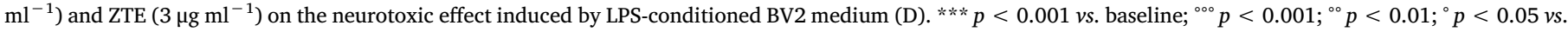
LPS-conditioned BV2 medium.

currently available regarding NP. This is the first study to support the analgesic effectiveness of a standardized $Z$. officinale extract in a model of peripheral mono-neuropathy, making it a promising candidate for the management of neuropathies. Indeed, contrary to the majority of herbal medicines, which often need repeated administration to achieve the pharmacological efficacy, ZOE reduced pain hypersensitivity after a single oral administration. This effect was characterized by a rapid onset of action, and it persisted up to $3 \mathrm{~h}$ after oral administration. This rapid but long-lasting effect may be related to the favourable pharmacokinetic profile of ZOE main constituents (Mukkavilli et al., 2017). At the same time, compared to conventional drugs used in the management of NP, ZOE did not induce tolerance in mice after repeated administration. Importantly, in contrast to first line therapy for NP [(e.g., pregabalin, which induces somnolence, altered motor coordination and ataxia (Verma et al., 2014)], ZOE did not cause any alteration in locomotor behaviour, confirming the safety profile of ginger. These important features may contribute to the amelioration of the NP patients quality of life, enhancing the therapeutic compliance.

Increasing evidence has emerged on the role of neuroinflammation in the pathogenesis of NP. In particular, traumatic damages to the peripheral nerve lead to a robust inflammatory response which may be involved in the initiation and maintenance of pain hypersensitivity. Indeed, microglia activation in the spinal cord is involved in synaptic alterations and can lead to nerve injuries and the insurgence of hypersensitivity. To better elucidate the mechanism of action of ginger, we tested ZOE in an in vitro model of neuroinflammation. LPS-stimulated BV2 cells were used as a validated and in vitro model for reproducing the effect of microglia activation in animals and they have been used to investigate the role of neuroinflammation in models of neuropathic pain (Masuda et al., 2014). One of the most important key effectors in signal transduction cascade associated with NP are MAPKs
(Edelmayer et al., 2014). Following nerve injury in the dorsal horn of the spinal cord, activation of MAPKs pathways occurs not only in neurons but also in glial cells showing a dominant role of microglia in the development and maintenance of NP (Ji et al., 2009). Thus, to investigate the analgesic mechanism of action of $\mathrm{ZOE}$, we evaluated whether the ZOE could modulate MAPKs activation. Our findings indicate a prominent involvement of the ERK pathway in the ZOE cellular action, as demonstrated by the drastic reduction of ERK1 and ERK2 increased phosphorylation in LPS-stimulated BV2 cells. Consistently with these results, ZOE reduced ERK activation in the ipsilateral side of the SNI spinal cord, in a similar manner to U0126. Numerous studies have reported that MEK1/2 inhibitors reduce ERK activation, leading to an attenuation of the symptoms associated with NP (PopiolekBarczyk et al., 2014). For these reasons, ZOE-induced inhibition of ERK1/2 activation may represent a main point of its analgesic effect. To further understanding the mechanism of action of ZOE, we also evaluated the effect on JNK1 protein levels in spinal cord. It has been shown that, after nerve injury, JNK1 preferentially localizes in spinal astrocytes and its inhibition leads to the reduction of mechanical allodynia (Zhuang et al., 2006). ZOE reduced pJNK1 protein levels in the ipsilateral side of spinal cord likely contributing to the analgesic effect of ginger.

Conversely, p38 appears to play a marginal role since no modification on pp38 levels was produced in BV2 cells. These data were further corroborated by the lack of ZOE effect on pp38 levels in SNI spinal cord following both acute and repeated oral administration. The prominent modulation of ERK signalling cascade represents a clinical advantage. Indeed, different MAPKs subtypes play different roles in the development of NP, such as specific inhibition of specific class of MAPKs produces positive effects at different stages of the clinical course of the pathology. ERK over-phosphorylation following nerve injury 
occurs first in neurons, microglia and, at later stages, in astrocytes in the ipsilateral dorsal horn. Taking into account that the interaction between glial and neuronal cells is key for the pathogenesis of pain, the modulation of ERK activation should be effective in the control of pain hypersensitivity at both early and later stages (Zhuang et al., 2005).

HDACs control the expression of a plethora of genes involved in pain perception and they are an emerging target for the control of algesia. Recently, we investigated the role of histone deacetylation in a model of NP showing an increase of HDAC1 protein expression in the ipsilateral side of the spinal cord in animal models of mononeuropathy. The reduction of HDAC1 over-expression by the administration of a specific HDAC1 inhibitor attenuated pain hypersensitivity (Sanna et al., 2017). Consistently, we showed that ZOE strongly reduces HDAC1 levels both in LPS-exposed BV2 cells and in SNI spinal cord samples.

Class I HDAC inhibitors have been reported to cause a reduction of neuroinflammation by modulation of pro-inflammatory cytokine expression in LPS-stimulated BV2 cells (Durham et al., 2017). An important cellular event involved in the pro-inflammatory response is the activation of NF- $\mathrm{kBp} 65$ and the subsequent transcription of a program of pro-inflammatory genes (Shih et al., 2015). Several findings indicate that the inactivation of NF-kBp65 pathways is linked to the repression of HDAC1 levels (Lee et al., 2017).

In addition, the activation of NF-kBp65 pathway contributes to the onset of neuropathic symptoms. Indeed, the silencing of this pathway is a possible target to control NP in several animal models (Yuan et al., 2014). In the present work, we demonstrated that ZOE reduces the nuclear translocation of pp65 and reverts the effect on IKBA $\alpha$ induced by LPS in BV2 cells. These results have been confirmed in SNI spinal cord samples, where ginger reduced the total expression level of pp65 after both acute and repeated administration. Furthermore, NF-кBp65 activation in microglia, by promoting transcription of pro-inflammatory mediators, may increase the production of reactive oxygen species and pro-inflammatory cytokines release (Shih et al., 2015). Consistently, ZOE significantly reduced the release of TNF- $\alpha$, IL- $1 \beta$ and IL- 6 in LPSstimulated BV2 cells. This effect was also observed in vivo, by the reduction of IL-1 $\beta$ protein levels in the ipsilateral side of the spinal cord of SNI mice. These data suggest that ZOE may attenuate neuroinflammation in NP conditions through the modulation of HDAC1 expression. To investigate the role of ZOE main constituents in its analgesic effect, we tested the effects produced by GIN, SHO and ZTE. We observed that GIN and SHO reduced ERK 1/2 phosphorylation, whereas ZTE was completely ineffective. Molecular docking simulations revealed a better binding mode for GIN and SHO in the active site of MEK1, compared to ZTE constituents, confirming the observed experimental activity. Gingerols and shogaols have been widely studied for their anti-inflammatory properties, particularly regarding the reduction of NFкBp65 activation and pro-inflammatory cytokines release from glial cells. Surprisingly, no effects on HDAC1, IKBA $\alpha$ and cytokines release were observed following GIN and SHO treatment. This discrepancy might be explained by taking into account that the effects on pro-inflammatory mediators are usually observed at concentrations 10-20 fold higher than those used in the present study (Lee et al., 2017). These lower doses may be insufficient to produce significant effects on the inflammatory pathways. ZTE showed opposite effects to GIN and SHO also towards the release of pro-inflammatory mediators. This volatile fraction largely decreased HDAC1 expression, NF- $\mathrm{\kappa B}$ activation and proinflammatory cytokines release. Coherently with recent studies on zerumbone, a sesquiterpenoid isolated from Zingiber zerumbet (L.) Roscoe rhizome essential oil (Chung et al., 2008) our results further support the efficacy of ginger sesquiterpenoids on HDAC1 modulation.

It is well known that the neuroinflammation plays a key role in the pathogenesis of NP. Currently, the communication between glial cells and neurons is an essential point for the development of novel therapies. After peripheral nerve injury, the microglia phenotype markedly changes to a pro-inflammatory phenotype which releases factors altering the normal neuronal activity. This condition seems to be involved in the initiation and maintenance of persistent NP (Tozaki-Saitoh and Tsuda, 2019). By treating SH-SY5Y neuronal cells with LPS-conditioned medium from ZOE-treated BV2 cells, we demonstrated that the antiinflammatory effect of ZOE may prevent the induction of neurotoxicity caused by microglia hyper-activation. Similar results were obtained with its main constituents, that all produced a partial beneficial effect in comparison with the complete protection produced by the phytocomplex. Thus, our results highlight ZOE as an interesting multi-target analgesic drug candidate for the management of NP.

\section{Conclusions}

In conclusion, we demonstrated that the acute and repeated oral administration of ZOE (200 $\mathrm{mg} \mathrm{kg}^{-1}$ ) reduced SNI-induced NP symptoms. These effects were linked to the reduction of pERK and HDAC1 expression, leading to the inhibition of NF- $\mathrm{\kappa B}$ activation and cytokines release. Oral administration of ZOE might represent an innovative and interesting perspective in the management of NP-related conditions.

\section{CRediT authorship contribution statement}

Vittoria Borgonetti: Investigation, Methodology, Formal analysis, Writing - original draft. Paolo Governa: Investigation, Writing - original draft. Marco Biagi: Writing - review \& editing. Federica Pellati: Investigation, Writing - review \& editing. Nicoletta Galeotti: Conceptualization, Methodology, Formal analysis, Writing - original draft, Writing - review \& editing.

\section{Declaration of Competing Interest}

The authors declared no conflict of interest.

\section{Acknowledgements}

This work was supported by grants from the University of Florence.

\section{Supplementary materials}

Supplementary material associated with this article can be found, in the online version, at doi:10.1016/j.phymed.2020.153307.

\section{References}

Adams, R.P., 2007. Identification of Essential Oil Components By Gas Chromatography/ Mass Spectrometry. Allured Publ., Carol Stream, IL, USA.

Bourquin, A.F., Süveges, M., Pertin, M., Gilliard, N., Sardy, S., Davison, A.C., Spahn, D.R., Decostered, I, 2006. Assessment and analysis of mechanical allodynia-like behavior induced by spared nerve injury (SNI) in the mouse. Pain 12214 , e1-14.

Charan, J., Kantharia, N., 2013. How to calculate sample size in animal studies? J. Pharmacol. Pharmacother 4, 303-306.

Choi, J.G., Kim, S.Y., Jeong, M., Oh, M.S., 2018. Pharmacotherapeutic potential of ginger and its compounds in age-related neurological disorders. Pharmacol. Ther. 182, 56-69.

Chung, I.M., Kim, M.Y., Park, W.H., Moon, H.I., 2008. Histone deacetylase inhibitors from the rhizomes of Zingiber zerumbet. Pharmazie 63, 774-776.

Curtis, M.J., Alexander, S., Cirino, G., Docherty, J.R., George, C.H., Giembycz, M.A., Hoyer, D., Insel, P.A., Izzo, A.A., Ji, Y., MacEwan, D.J., Sobey, C.G., Stanford, S.C., Teixeira, M.M., Wonnacott, S., Ahiuwalia, A., 2018. Experimental design and analysis and their reporting II: updated and simplified guidance for authors and peer reviewers. Br. J. Pharmacol. 175, 987-993.

Durham, B.S., Grigg, R., Wood, I.C., 2017. Inhibition of histone deacetylase 1 or 2 reduces induced cytokine expression in microglia through a protein synthesis independent mechanism. J. Neurochem. 143, 214-224.

Edelmayer, R.M., Brederson, J.D., Jarvis, M.F., Bitner, R.S., 2014. Biochemical and pharmacological assessment of MAP-kinase signaling along pain pathways in experimental rodent models: a potential tool for the discovery of novel antinociceptive therapeutics. Biochem. Pharmacol. 87, 390-398.

EMA, European Medicines Agency2012. Assessment report on Zingiber officinale Roscoe, rhizoma. Available at:www.ema.europa.eu/en/documents/herbal-report/finalassessment-report-zingiber-officinale-roscoe-rhizoma_en.pdf.

Ferguson, N.M., 1956. A Textbook of Pharmacognosy. Macmillan, London.

Forouzanfar, F., Hosseinzadeh, H., 2018. Medicinal herbs in the treatment of neuropathic 
pain: a review. Iran. J. Basic Med. Sci. 21, 347-358.

Hargreaves, K., Dubner, R., Brown, F., Flores, C., Joris, J., 1988. A new and sensitive method for measuring thermal nociception in cutaneous hyperalgesia. Pain 32 $77-88$.

Jensen, T.S., Finnerup, N.B., 2014. Allodynia and hyperalgesia in neuropathic pain: clinical manifestations and mechanisms. Lancet Neurol. 13, 924-935.

Ji, R.R., Gereau IV, R.W., Malcangio, M., Strichartz, G.R., 2009. MAP kinase and pain. Brain Res. Rev. 60, 135-148.

Kilkenny, C., Browne, W.J., Cuthill, I.C., Emerson, M., Altman, D.G., 2010. Improving bioscience research reporting: the ARRIVE guidelines for reporting animal research. PLoS Biol. 8, e1000412.

Lee, C., Kim, B.G., Kim, J.H., Chun, J., Im, J.P., Kim, J.S., 2017. Sodium butyrate inhibits the NF-kappa B signaling pathway and histone deacetylation, and attenuates experimental colitis in an IL-10 independent manner. Int. Immunopharmacol. 51, $47-56$.

Masuda, T., Iwamoto, S., Yoshinaga, R., Tozaki-Saitoh, H., Nishiyama, A., Mak, T.W., Tamura, T., Tsuda, M., Inoue, K., 2014. Transcription factor IRF5 drives P2X4R + -reactive microglia gating neuropathic pain. Nat. Commun 5, 3771.

McGrath, J.C., Lilley, E., 2015. Implementing guidelines on reporting research using animals (ARRIVE etc.): new requirements for publication in BJP. Br. J. Pharmacol. 172, 3189-3193.

Mukkavilli, R., Yang, C., Tanwar, R.S., Ghareeb, A., Luthra, L., Aneja, R., 2017. Absorption, metabolic stability, and pharmacokinetics of ginger phytochemicals. Molecules 22 E553.

Popiolek-Barczyk, K., Makuch, W., Rojewska, E., Pilat, D., Mika, J., 2014. Inhibition of intracellular signaling pathways NF-KB and MEK1/2 attenuates neuropathic pain development and enhances morphine analgesia. Pharmacol. Rep. 66, 845-851.

Sanna, M.D., Guandalini, L., Romanelli, M.N., Galeotti, N., 2017. The new HDAC1 inhibitor LG325 ameliorates neuropathic pain in a mouse model. Pharmacol. Biochem. Behav. 160, 70-75.

Sanna, M.D., Les, F., Lopez, V., Galeotti, N., 2019. Lavender (Lavandula angustifolia Mill.) essential oil alleviates neuropathic pain in mice with spared nerve injury. Front. Pharmacol 10, 472
Sanna, M.D., Stark, H., Lucarini, L., Ghelardini, C., Masini, E., Galeotti, N., 2015. Histamine $\mathrm{H} 4$ receptor activation alleviates neuropathic pain through differential regulation of ERK, JNK, and P38 MAPK phosphorylation. Pain 156, 2492-2504.

Semwal, R.B., Semwal, D.K., Combrinck, S., Viljoen, A.M., 2015. Gingerols and shogaols: Important nutraceutical principles from ginger. Phytochemistry 117, 554-568.

Shih, R.H., Wang, C.Y., Yang, C.M., 2015. NF-kappaB signaling pathways in neurological inflammation: a mini review. Front. Mol. Neurosci 18, 1-8.

Singh, H., Bhushan, S., Arora, R., Singh Buttar, H., Arora, S., Singh, B., 2017. Alternative treatment strategies for neuropathic pain: role of Indian medicinal plants and compounds of plant origin - a review. Biomed. Pharmacother. 92, 634-650.

Subedi, L., Lee, J.H., Yumnam, S., Ji, E., Kim, S.Y., 2019. Anti-inflammatory effect of sulforaphane on LPS-activated microglia potentially through JNK/AP-1/NF-KB inhibition and Nrf2/HO-1 activation. Cells 8, 194.

Sun, J., Chen, F., Braun, C., Zhou, Y.Q., Rittner, H., Tian, Y.K., Cai, X.Y., 2018. Role of curcumin in the management of pathological pain. Phytomedicine 48, 129-140.

Tozaki-Saitoh, H., Tsuda, M., 2019. Microglia-neuron interactions in the models of neuropathic pain. Biochem. Pharmacol. 169, 113614

Verma, V., Singh, N., Singh Jaggi, A., 2014. Pregabalin in neuropathic pain: evidences and possible mechanisms. Curr. Neuropharmacol. 12, 44-56.

Yuan, B., Liu, D., Liu, X., 2014. Spinal cord stimulation exerts analgesia effects in chronic constriction injury rats via suppression of the TLR4/NF-kB pathway. Neurosci. Lett. 581, 63-68.

Zhao, S., Pi, C., Ye, Y., Zhao, L., Wei, Y., 2019. Recent advances of analogues of curcumin for treatment of cancer. Eur. J. Med. Chem. 180, 524-535.

Zhuang, Z.Y., Gerner, P., Woolf, C.J., Ji, R.R., 2005. ERK is sequentially activated in neurons, microglia, and astrocytes by spinal nerve ligation and contributes to mechanical allodynia in this neuropathic pain model. Pain 114, 149-159.

Zhuang, Z.Y., Wen, Y.R., Zhang, D.R., Borsello, T., Bonny, C., Strichartz, G.R., Decostered, I., Ji, R.R., 2006. A peptide c-Jun N-terminal kinase (JNK) inhibitor blocks mechanical allodynia after spinal nerve ligation: respective roles of JNK activation in primary sensory neurons and spinal astrocytes for neuropathic pain development and maintenance. J. Neurosci. 26, 3551-3560. 